\begin{tabular}{|c|l|}
\hline Title & $\begin{array}{l}\text { Combination of sensitivity-based and random sampling-based methodologies for efficient uncertainty quantification } \\
\text { calculations with control variates method }\end{array}$ \\
\hline Author(s) & Nihira, Shunsuke; Chiba, Go \\
\hline Citation & $\begin{array}{l}\text { Journal of nuclear science and technology, 56(11), 971-980 } \\
\text { https://doi.org/10.1080/00223131.2019.1630022 }\end{array}$ \\
\hline Issue Date & 2019 \\
\hline Doc URL & http://hdl.handle.net/2115/79642 \\
\hline Rights & $\begin{array}{l}\text { This is an A ccepted Manuscript of an article published by Taylor \& Francis in Journal of nuclear science and } \\
\text { technology on Nov. 2019, available online: http:/www.tandfonline.com/10.1080/00223131.2019.1630022. }\end{array}$ \\
\hline Type & article (author version) \\
\hline File Information & paper.pdf \\
\hline
\end{tabular}

Instructions for use 


\title{
Combination of sensitivity-based and random sampling-based methodologies for efficient uncertainty quantification calculations with control variates method
}

\author{
Shunsuke Nihira ${ }^{1}$, Go Chiba ${ }^{1 *}$ \\ ${ }^{1}$ Hokkaido University, Kita-ku, Sapporo 060-8628, Japan
}

A combined method of the sensitivity-based and random sampling-based methodologies is proposed for efficient uncertainty quantification calculations. The proposed method is based on the control variates $(\mathrm{CV})$ method, in which a mean value of a target parameter can be estimated efficiently with a help of a mockup parameter whose mean value is well known. Standard deviations can be also efficiently estimated from two mean values of stochastic parameters; a target parameter itself and its square. In the present work, the CV method is applied to a toy problem, in which a linear approximation to a target parameter is regarded as a mockup parameter. This case corresponds to our proposed method to combine the sensitivity-based and random sampling-based methodologies. Numerical results reveal that the proposed method efficiently works. As a preliminary test of application of our proposed method to realistic problems, nuclear fuel burnup calculations are considered, and uncertainties of nuclides number densities after burnup are calculated. Uncertainties of number densities of cesium-134 and europium-151 are calculated by the proposed method, and it is demonstrated that we can carry out uncertainty quantification calculations more efficiently with our proposed method than with the normal random sampling method. 
Keywords: uncertainty quantification, random sampling, sensitivity, control variates method 


\section{Introduction}

Uncertainty quantification in numerical simulation has been one of important research subjects in the field of reactor physics, and various works especially about nuclear datainduced uncertainties have been conducted so far. There are mainly two methodologies for nuclear data-induced-uncertainty quantification; one is a sensitivity-based methodology and the other is a random sampling-based methodology. The sensitivity-based methodology was mainly adopted in the past, but as computer power has become significant, the random sampling-based methodology, which is based on the Monte Carlo method, now becomes a standard method in uncertainty quantification calculations. The random sampling method to estimate statistical quantities of target parameters is very simple and can be applied to various problems[1,2]. One of great advantages of the random sampling-based methodology is that it can handle with non-linear problems, which cannot be with the sensitivity-based methodology. On the other hand, the random sampling-based methodology requires high computational cost to obtain numerical results with small statistical uncertainties. Reduction technique of these statistical uncertainties inherent to the random sampling method is important, and several efficient sampling methods such as the Latin hypercube sampling have been proposed[1].

The control variates $(\mathrm{CV}) \operatorname{method}[3]$ is one of variance reduction methods in Monte Carlo calculations. With this method, one can efficiently estimate a mean value of a target parameter by using another parameter whose mean value is well known and is highly correlated to the target parameter. Efficiency of the CV method depends on this similarity, and high efficiency is expected when one can prepare a parameter whose similarity to the target parameter is high.

In the field of reactor physics, an attempt about application of the CV method to uncertainty quantification calculations were initially made by Shiba, Iwahashi and other co-authors $[4,5]$ as far as the present authors' knowledge. They have shown that both the 
mean values and standard deviations can be accurately estimated from a small number of samples by adopting the CV method. Numerical results about infinite multiplication factors in fresh and burned fuel assemblies and number density of plutonium-239 in burned nuclear fuels have been presented, and it has been demonstrated that efficient estimation of uncertainties of these parameters/quantities become possible if parameters similar to the target parameters can be prepared.

This work about the application of the CV method to uncertainty quantification calculations has led us to a new idea; uncertainty quantification calculations with the random sampling-based methodology can be accelerated by use of results of the sensitivity-based methodology by adopting the CV method. Based on this idea, the present paper newly proposes a combined method of the random sampling-based and sensitivity-based methodologies for efficient uncertainty quantification calculations, and presents some results of simple toy problems and preliminary test calculations for realistic problems.

The present paper is organized as follows. In Section 2, the fundamental theory of the CV method is described. Detailed procedure to estimate standard deviations of stochastic parameters from a finite number of samples with the CV method has not been presented in the previous works $[4,5]$, so it is described in this section. In Section 3, numerical results of toy problems and nuclear fuel depletion problems are presented, and finally the present study is concluded in Section 4.

\section{Theory of control variates method}

\subsection{Mean value estimation}

Here let us consider a stochastic parameter $X$ (a target parameter), which we attempt to estimate its mean value, and consider another stochastic parameter $Y$ (a mockup parameter) whose mean value and variance are well known and is highly correlated to $X$. Also, let mean value and variance of $X$ denoted to as $E[X]$ and $V[X]$. Remember that 
$E[Y]$ and $V[Y]$ are assumed well known.

Now consider a parameter $H$ which is defined as $H=X-\alpha Y$. A mean value of $H$, $E[H]$, can be written as

$$
E[H]=E[X]-\alpha E[Y]
$$

This equation suggests that $E[X]$ can be efficiently estimated as $E[H]+\alpha E[Y]$ if variance of $H, V[H]$, is adequately small because variance of $E[H]$ is proportional to $V[H]$. $V[H]$ can be expressed by using covariance between $X$ and $Y$, $\operatorname{Cov}[X, Y]$, and $V[X]$ as

$$
V[H]=V[X-\alpha Y]=V[X]+\alpha^{2} V[Y]-2 \alpha \operatorname{Cov}[X, Y]
$$

and a condition of minimization of $V[H]$ can be given as

$$
\frac{\partial V[H]}{\partial \alpha}=0 .
$$

From the above equations, we can derive

$$
\alpha=\frac{\operatorname{Cov}[X, Y]}{V[Y]}
$$

When we use the above $\alpha, V[H]$ can be written as

$$
V[H]=V[X]-\frac{\operatorname{Cov}[X, Y]}{V[Y]}=\left(1-\operatorname{Corr}[X, Y]^{2}\right) V[X],
$$

where $\operatorname{Corr}[X, Y]$ is a correlation coefficient between $X$ and $Y$. Since square of a correlation coefficient must be in a range of $[0,1], V[H]$ is smaller than $V[X]$ if the correlation coefficient is non-zero. This means that statistical uncertainty of $E[H]$ should be smaller than $E[X]$, so we can efficiently estimate $E[X]$ from $E[H]$ instead of estimating $E[X]$ directly.

A procedure to estimate a mean value by the CV method can be described as follows:

(1) Let us assume that $E[Y]$ and $V[Y]$ are well known.

(2) A finite number of samples of $X$ and $Y$ are prepared.

(3) From these samples of $X$ and $Y$, covariance between $X$ and $Y$ is calculated and $\alpha$ is obtained with Equation (4). Here we have two options about $V[Y]$; a rigorous 
value and an estimated value from the samples. In the present study, we will use the estimated value for $V[Y]$ to keep consistency between $\operatorname{Cov}[X, Y]$ and $V[Y]$. This will be discussed later.

(4) Samples about $H=X-\alpha Y$ are calculated, and from these samples $E[H]$ is calculated.

(5) $E[X]$ is estimated as $E[H]+\alpha E[Y]$.

\subsection{Variance estimation}

The CV method can be applied not only to mean value estimation but also to variance estimation as follows. Variance of $X$ can be presented with mean values of $X$ and $X^{2}$ as

$$
V[X]=E\left[X^{2}\right]-(E[X])^{2}
$$

Now we know that estimation of a mean value of any stochastic parameters can be efficiently carried out with the CV method, so this concept can be directly applied to variance estimation based on Eq. (6). In order to estimate $E\left[X^{2}\right]$ by the CV method, we define $\bar{H}$ as

$$
\bar{H}=X^{2}-\beta Y^{2},
$$

and a mean value of $X^{2}$ can be written as

$$
E\left[X^{2}\right]=E[\bar{H}]+\beta E\left[Y^{2}\right]
$$

The coefficient $\beta$ is defined so as to minimize $V[\bar{H}]$ as

$$
\beta=\frac{\operatorname{Cov}\left[X^{2}, Y^{2}\right]}{V\left[Y^{2}\right]}
$$

A procedure to estimate variance by the CV method can be described as follows:

(1) Let us assume that $E[Y]$ and $V[Y]$ are well known. In this situation, $E\left[Y^{2}\right]$ is also well known because $E\left[Y^{2}\right]=V[Y]+(E[Y])^{2}$.

(2) A finite number of samples of $X$ and $Y$ are prepared. 
(3) From these samples of $X$ and $Y$, covariance between $X^{2}$ and $Y^{2}$ is estimated and $\beta$ is obtained with Equation (9). Here we have to use $V\left[Y^{2}\right]$ estimated from the samples because we do not know a rigorous value of $V\left[Y^{2}\right]$ generally.

(4) Samples about $\bar{H}=X^{2}-\beta Y^{2}$ are calculated, and from these samples $E[\bar{H}]$ is calculated.

(5) $E\left[X^{2}\right]$ is obtained with Equation (8), and finally $V[X]$ can be estimated as $V[X]=$ $E\left[X^{2}\right]-(E[X])^{2}$, where $E[X]$ is estimated also with the CV method.

\subsection{Use of results by sensitivity-based methodology as mockup parameters}

As described in the preceding sections, we are now focusing on quantifications of nuclear data-induced uncertainties in reactor physics parameters. Under this circumstance, input stochastic parameters are nuclear data and output stochastic parameters are reactor physics parameters. In the sensitivity-based methodology, linearity is assumed between input stochastic parameters to output stochastic parameters, but generally this linearity is not guaranteed in every reactor physics problems. However, when we consider fictitious output stochastic parameters which linearly depend on input parameters, these fictitious parameters can be good mockup parameters of the output parameters. With sensitivity coefficients of output parameters with respect to input parameters, mean values and standard deviations of the fictitious output parameters can be accurately calculated with the uncertainty propagation formula, and also calculations of fictitious output parameters from samples can be carried out quite easily without any computational burden. Here we propose to use these fictitious parameters in the CV method to perform efficient estimations of statistical quantities of target stochastic parameters. 


\section{Numerical results}

\subsection{Toy problem of correlated two parameters}

Here we consider two stochastic parameters $X$ and $Y$. These are described by the Gaussian distribution and correlated to each other. Mean values and standard deviations are determined as $(0.5,0.25)$ for $X$, and $(1.0,0.5)$ for $Y$. In order to adopt the CV method, $X$ is regarded as a target parameter whose statistical quantities are unknown, and $Y$ is a mockup parameter whose statistical quantities are well known. A correlation coefficient between $X$ and $Y$ is assumed 0.95. Statistical quantities such as a mean value and a standard deviation of $X$ are estimated from a finite number of samples. Uncertainties of the estimated statistical quantities are calculated from 100 sets of estimated statistical quantities; 100 sets of independent random sampling calculations with a certain number of samples to estimate statistical quantities are carried out. Figures $\mathbf{1}$ and $\mathbf{2}$ show mean value and standard deviation of $X$ estimated with the normal random sampling method and the CV method. Error bars indicate $1 \sigma$ uncertainties of estimated statistical quantities.

[Figure 1 about here.]

[Figure 2 about here.]

In both the mean value and the standard deviation, uncertainties of these estimations become small if the number of samples increases, and uncertainties of these estimations by the CV method is smaller than those by the normal random sampling method. This shows effectiveness of the CV method.

As mentioned in the preceding section, we have another choice about $V[Y]$ in calculating $\alpha$. As a default procedure, we utilize $V[Y]$ estimated from samples to keep consistency to $\operatorname{Cov}[X, Y]$ which is numerator of $\alpha$, but it is also possible to use the rigorous value of $V[Y]$. Here calculations with this different option for $\alpha$ calculations are carried out. To quantify the efficiency of the CV method, a new quantity, uncertainty reduction, is defined 
as a ratio of uncertainty of estimations by the CV method to that by the normal random sampling method. Results are shown in Figure 3.

[Figure 3 about here.]

A case 1 is the default case, and a case 2 is the case with the other option for $\alpha$ calculations. It is clearly shown that the case 1 gives much smaller uncertainties than the case 2 , especially when the number of samples is small. Further deep investigation about this still remains as a future subject, but in the following calculations, we will continue to use the default procedure: the case 1.

Next, dependence of uncertainty reduction on correlation between a target parameter and a mockup parameter is investigated. Figure 4 shows uncertainty reduction under the same numerical conditions with different correlation coefficients.

[Figure 4 about here.]

These figures tell us two things. One is that uncertainty reduction strongly depends on correlation coefficients. This result suggests that it is necessary to choose a mockup parameter whose correlation to a target parameter is at least around 0.9 . The other is that uncertainty reduction doesn't depend on the number of samples. This means that we can grasp performance of the CV method by using results obtained only from a certain number of samples.

In the above calculations, mean values and standard deviations of $X$ and $Y$ are determined so as to give the same relative standard deviation. Here we examine dependence of uncertainty reduction on mean values and standard deviations of $X$ and $Y$.

We change a mean value and a standard deviation of $X$ in the ranges of $[0.25,0.75]$ and $[0.125,0.375]$, respectively, and the same calculations are carried out. Figure 5 shows uncertainty reduction of standard deviations of $X$. It is shown that uncertainty reduction effect generally becomes small when a mean value and a standard deviation of $X$ become apart from their original values. The same calculations are conducted when a mean value 
and a standard deviation of $Y$ are changed in the ranges of $[0.5,1.5]$ and $[0.25,0.75]$, respectively. Figure 6 shows uncertainty reduction of a standard deviation of $X$ in these calculations, and the similar trend with Figure 5 can be observed.

[Figure 5 about here.]

[Figure 6 about here.]

The above results are re-plotted with relative difference between relative standard deviations of $X$ and $Y$ in Figure 7 . Generally it can be said that uncertainty reduction significantly depends on difference in relative standard deviations between target and mockup parameters.

[Figure 7 about here.]

\subsection{Toy problem for the CV method with linear approximation results as mockup parameters}

Here we consider two stochastic parameters $X$ and $Y$, and those depend on a common stochastic parameter $Z$ which is described by the Gaussian distribution and whose mean value and standard deviation are 1.0 and 0.5 , respectively. When a sample of $Z$ is presented as $z$, corresponding samples of $X$ and $Y, x$ and $y$, are written as

$$
\begin{aligned}
& x=z+S z^{2}, \\
& y=z .
\end{aligned}
$$

To adopt the CV method to this problem, we assume that $X$ is a target parameter and $Y$ is a mockup parameter, so a mean value and a standard deviation of $Y$ are assumed well known. It is expected that the performance of the CV method depends on $S$ in Eq. (10), so calculations with the CV method are carried out with different values of $S$. Frequency distribution functions of $X$ with different values of $S$ based on results with a large number of samples are shown in Figure 8. It is clearly shown that the frequency distribution is 
distorted when the second-order effect, a value of $S$, is large.

[Figure 8 about here.]

A standard deviation of $X$ is estimated from a finite number of samples with the normal random sampling method and the $\mathrm{CV}$ method in which a mockup parameter is $Y$. Uncertainty of the estimated standard deviations are calculated from 100 sets of random sampling calculations with a certain number of samples as the preceding section. Figure 9 shows standard deviations in the case of $S=0.2$. Significant reduction of the uncertainty in estimated standard deviations by the CV method is observed. Figure 10 shows uncertainty reduction of standard deviations with different values of $S$. As the second-order effect becomes large, uncertainty reduction effect becomes small, but it still be around 0.2 even though $S$ is 0.2 . Correlation coefficients between $X$ and $Y$ are 0.999 , 0.998 and 0.995 in the cases of $S=0.05,0.1$ and 0.2 .

[Figure 9 about here.]

[Figure 10 about here.]

In the above calculations, uncertainties of estimated statistical quantities are calculated through 100 sets of random sampling calculations, but this approach becomes impractical when we adopt the $\mathrm{CV}$ method to realistic problems in which calculations of a target parameter require heavy computational burden. Here we use the bootstrap method in order to simply estimate uncertainties of statistical quantities only from one set of random sampling calculations $[6,7]$. In the present work, we apply the following simple procedure based on the bootstrap method; when we estimate uncertainties of statistical quantities obtained from $N$ samples, $N$ bootstrap replicates, which are chosen randomly from the original $N$ samples, are generated, and $E[X], E\left[X^{2}\right]$ and $V[X]$ are calculated from these $N$ samples. This calculation is performed 1,000 times, so 1,000 sets of the bootstrap replicates are generated, and 1,000 sets of $E[X]$ and $V[X]$ are obtained. Standard deviations calculated from these 1,000 sets of $E[X]$ and $V[X]$ can be approxi- 
mately regarded as standard deviations of $E[X]$ and $V[X]$ estimated with the CV method from $N$ samples. With this procedure, we can roughly estimate uncertainties of estimated statistical quantities without direct calculations of additional sets of samples.

Uncertainties of estimated standard deviations in the toy problem of this subsection are estimated by this simple bootstrap method and results are compared with reference ones, which are calculated from 100 sets of statistical quantities estimated from random sampling calculations. Because uncertainties obtained with the simple bootstrap method should include uncertainties, estimations by this method are carried out three times with different random seeds. Results of standard deviations of estimated sample standard deviations are shown in Figure 11 with different values of $S$. These figures suggest that our bootstrap procedure tends to underestimate uncertainties of estimated statistical quantities if the number of samples is less than 1,000, but it can roughly estimate these uncertainties if the number of samples is over 1,000. This bootstrap procedure will be adopted to calculations in the following section.

[Figure 11 about here.]

\subsection{Preliminary application to nuclear fuel burnup problems}

Finally the proposed method to combine the sensitivity-based and random samplingbased methodologies is applied to nuclear fuel burnup problems in this section. This is just a preliminary test, and extensive application test is required in future.

Nuclides number densities after burnup of $45 \mathrm{GWd} / \mathrm{t}$ of a $\mathrm{UO}_{2}$ fuel pin-cell with uranium-235 enrichment of $4.1 \mathrm{wt} \%$ are concerned, and uncertainties of nuclides number densities induced by nuclear data are estimated by the proposed method. Nuclear fuel burnup calculations are carried out with a reactor physics code system CBZ, which is under development at Hokkaido University. Verification of CBZ against nuclear fuel burnup calculations has been well demonstrated in our previous work[8]. Sensitivities of nuclides 
number densities after burnup with respect to nuclear data are calculated by the depletion perturbation theory capability of CBZ[9]. Fission yields data and radioactive decay data of fission product (FP) nuclides are taken from an updated version of JENDL FP yields data file-2011(JENDL/FPY-2011) and FP decay data file-2011(JENDL/FPD-2011)[10], in which nuclear data of 1,400 FP nuclides are evaluated. Covariance data on decay halflives, decay branching ratios and independent fission yields provided in these files are considered in our calculations. Correlations in fission yields among FP nuclides belonging to the same mass chain are considered by the method presented in the reference[10]. One-hundred percent standard deviation is assumed to nuclear data to which covariance data are not provided in these files. Capture, fission and $(\mathrm{n}, 2 \mathrm{n})$ reaction cross section uncertainties of twenty important actinoid nuclides, which are given in JENDL-4.0[11], are also considered.

In this calculation, relative perturbation amounts of half-lives, decay branching ratios, independent fission yields and reaction cross sections are sampled based on their covariance matrices and these nuclear data are changed according to these perturbation amounts. A simplified burnup nuclide chain consisting of $138 \mathrm{FP}$ nuclides is generated from these perturbed nuclear data to reduce computational time, a fuel depletion calculation with this simplified chain is carried out, and finally samples about concerned quantities are obtained. In this random sampling process, negative values of nuclear data and negative number densities are allowed. At the same time, these perturbation amounts of nuclear data are used to calculate mock-up samples of concerned quantities. Here a relative perturbation amount of nuclear data $i, \sigma_{i}$, is denoted to as $\delta_{i}$. Based on a linear assumption, a mock-up sample of a concerned quantity $p$ can be calculated as

$$
p=\bar{p}+\sum_{i}\left(\frac{d p}{d \sigma_{i}} \cdot \frac{\sigma_{i}}{p}\right) \delta_{i},
$$

where $\bar{p}$ is an original value calculated from unperturbed nuclear data and $\left(\frac{d p}{d \sigma_{i}} \cdot \frac{\sigma_{i}}{p}\right)$ is a sensitivity of $p$ with respect to $\sigma_{i}$. 
In this preliminary calculation, uncertainties of number densities of cesium-134 and europium-151 are concerned. Cesium-134 is chosen because of its importance in shortterm decay heat, and europium-151 is chosen because non-linear effect on nuclear data has been observed in our previous study[12]. Relative standard deviations of number densities of these two nuclides after burnup of $45 \mathrm{GWD} / \mathrm{t}$ calculated with the normal random sampling method and our proposed method are shown in Figures 12 and 13. Error bars indicate $1 \sigma$ uncertainties calculated by the simple bootstrap method. In addition, uncertainty reductions in these standard deviations are shown in Figure 14. In both the nuclides, uncertainty reduction can be attained by the proposed method and it is clearly shown that our proposed method is promising. The uncertainty reduction is more significant in the cesium-134 case than in the europium-151 case. This can be explained by a difference in correlation coefficients between the rigorous model and the linear model; 0.999 in cesium-134 and 0.988 in europium-151. A difference in standard deviations of the europium-151 number densities is observed in the result with 10,000 samples between the normal sampling method and the proposed method. This difference is smaller than $2 \sigma$ of uncertainties estimated by the simple bootstrap method. If we consider ambiguity inherent to the bootstrap method and that caused by our simple procedure, this difference can be regarded reasonable at present.

[Figure 12 about here.]

[Figure 13 about here.]

[Figure 14 about here.]

\section{Conclusion}

A combined method of the sensitivity-based and random sampling-based methodologies has been proposed for efficient uncertainty quantification calculations. The proposed method is based on the CV method, in which a mean value of a target parameter can be 
estimated efficiently with a help of a mockup parameter whose mean value is well known. The application of the CV method to uncertainty quantification calculations has been carried out by the other research group, but its theoretical detail has not been presented, so it has been described in the present paper. Standard deviations can be efficiently estimated from two mean values of stochastic parameters; one is a target parameter itself and the other is its square. In the present work, the CV method has been applied to a toy problem with two stochastic parameters which are highly correlated to each other, and effectiveness of the CV method in uncertainty quantification calculations has been demonstrated. Then, the CV method has been applied to another toy problem, in which a linear approximation to a target parameter is regarded as a mockup parameter. This case corresponds to our proposed method to combine the sensitivity-based and random sampling-based methodologies. Numerical results have revealed that the proposed method has efficiently worked, and some parameter surveys have been carried out to understand the feature of the proposed method. Finally, as a preliminary test of application of our proposed method to realistic problems, nuclear fuel burnup calculations have been considered, and uncertainties of nuclides number densities after burnup have been calculated. Uncertainties induced by various types of nuclear data such as decay half-lives, decay branching ratios, fission yields and neutron-nuclide reaction cross sections have been taken into account. Uncertainties of number densities of cesium-134 and europium-151 have been calculated by the proposed method, and it has been demonstrated that we can carry out uncertainty quantification calculations more efficiently with our proposed method than with the normal random sampling method.

Through the present study, it can be concluded that our proposed method is promising. Extensive application tests of our proposed method to wide ranges of problems are necessary and are now under progress. This work includes adoption of more reliable bootstrap method than the present simple one, and trial to adopt much more simplified model, 
such as pin-cell model for fuel assembly model, instead of rigorous target model in mockup parameter calculations.

\section{Acknowledgement}

The authors greatly appreciate for Dr. Toshihisa Yamamoto of nuclear regulation authority of Japan, who has initially adopted the control variates method to uncertainty quantification calculations and has instructed this method to the authors.

\section{References}

[1] McKay MD. Uncertainty analysis. CRC Press; 1988. Chap. 4, Sensitivity and uncertainty analysis using a statistical sample of input values; p.145-186.

[2] Kawano T, Hanson KM, Frankle S, Talou P, Chadwick MB, Little RC. Evaluation and propagation of the ${ }^{239} \mathrm{Pu}$ fission cross-section uncertainties using a Monte Carlo technique. Nucl Sci Eng. 2006; 153:1-7.

[3] Krose DP, Taimre T, Botev ZI. Handbook of Monte Carlo Methods. New York: John Wiley \& Sons; 2011.

[4] Shiba S, Sakai T, Yamamoto T. Variance reduction on uncertainty analysis by random sampling method (1) Variance reduction approach to uncertainty analysis for infinite multiplication factor in fuel assembly. Proc. annual meeting of Atomic Energy Society of Japan; 2018 March 26-28; Osaka (Japan). [in Japanese]

[5] Iwahashi D, Sakai T, Yamamoto T. Variance reduction on uncertainty analysis by random sampling method (2) Variance reduction in burnup calculation. Proc. annual meeting of Atomic Energy Society of Japan; 2018 March 26-28; Osaka (Japan). [in Japanese]

[6] Efron B, Tibshirani R. Bootstrap methods for standard errors, confidence intervals, and other measures of statistical accuracy. Stat. Sci. 1986; 1:54-75.

[7] Endo T, Watanabe T, Yamamoto A. A confidence interval estimation by bootstrap method for 
uncertainty quantification using random sampling method/ J. Nucl. Sci. Technol. 2015; 52:993-999.

[8] Okumura S, Chiba G. Development of nuclear fuel depletion calculation capability for LWR fuel assembly in reactor physics code system CBZ. Proc. Reactor Physics Asia 2017; 2017 Aug 24-25; Chengdu (China).

[9] Chiba G, Kawamoto Y, Narabayashi T. Development of a fuel depletion sensitivity calculation module for multi-cell problem in a deterministic reactor physics code system CBZ. Ann Nucl Energy. 2016; 96: 277-286.

[10] Katakura J. JENDL FP decay data file 2011 and fission yields data file 2011. Japan: Japan Atomic Energy Agency; 2011, JAEA-Data/Code 2011-025.

[11] Shibata K, Iwamoto O, Nakagawa T, Iwamoto N, Ichihara A, Kunieda S, Chiba S, Furukawa K, Otuka N, Ohsawa T, Murata T, Matsunobu H, Zukaran A, Kameda S, Katakura J. JENDL-4.0: A new library for nuclear science and technology. J Nucl Sci Technol. 2011; 48:1-30.

[12] Nihira S, Chiba G. Uncertainty quantification of nuclides number densities after fuel depletion by random sampling method. Proc. Reactor Physics Asia 2017; 2017 Aug 24-25; Chengdu (China). 


\section{Figure Captions}

Figure 1 Mean values estimated from samples

Figure 2 Standard deviations estimated from samples

Figure 3 Uncertainty reduction in estimations of mean value and standard deviation

Figure 4 Dependence of uncertainty reduction in estimations of mean value and standard deviation on correlation coefficients $C$

Figure 5 Uncertainty reduction in estimations of standard deviation with different conditions for a target parameter

Figure 6 Uncertainty reduction in estimations of standard deviation with different conditions for a mockup parameter

Figure 7 Uncertainty reduction in estimations of standard deviation with relative difference in relative standard deviations between target and mockup parameters

Figure 8 Frequency distribution functions of target parameters with different value of $S$

Figure 9 Standard deviations for non-linear problem in the case of $S=0.2$

Figure 10 Uncertainty reduction of standard deviation estimated by the CV method for non-linear problem 
Figure 11 Standard deviation of estimated sample standard deviation calculated by the bootstrap method for non-linear system

Figure 12 Standard deviation of cesium-134 number density after burnup

Figure 13 Standard deviation of europium-151 number density after burnup

Figure 14 Uncertainty reduction in standard deviation of cesium-134 and europium-151 number density after burnup 
(a) Normal

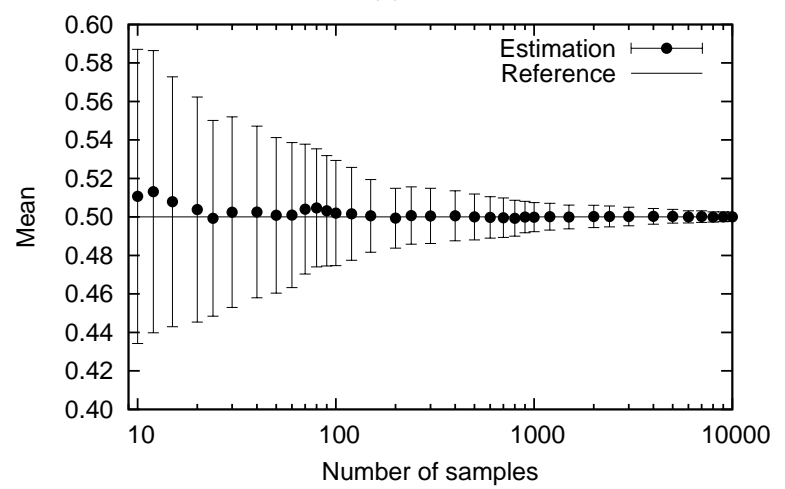

(b) $\mathrm{CV}$

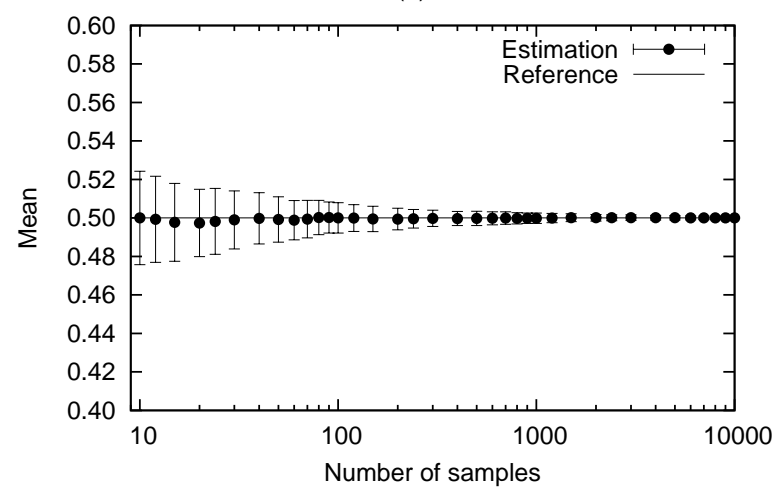

Figure 1 Mean values estimated from samples

S. Nihira and G. Chiba

Combination of sensitivity-based and random sampling-based methodologies for efficient uncertainty quantification calculations with control variates method 
(a) Normal

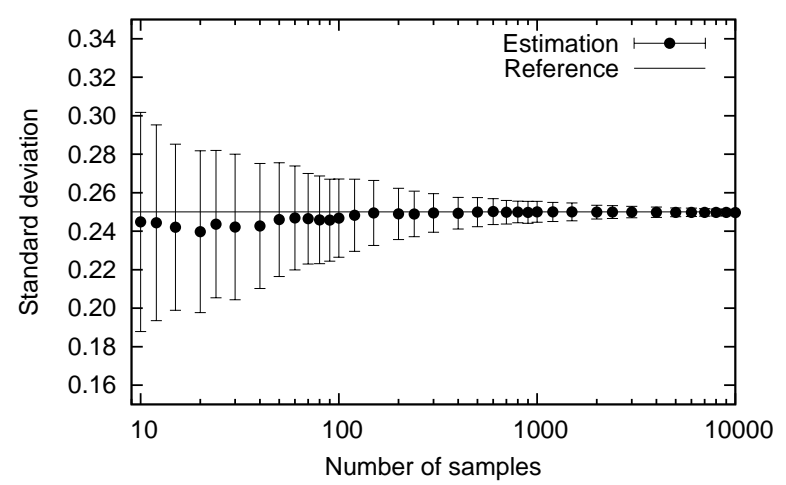

(b) $\mathrm{CV}$

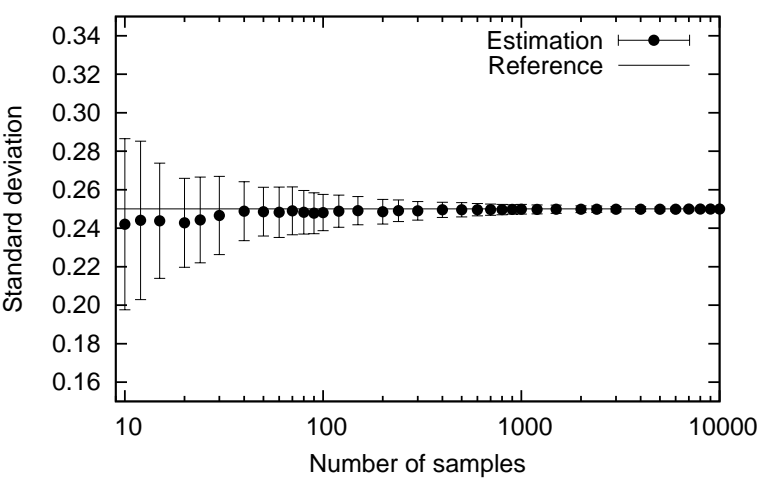

Figure 2 Standard deviations estimated from samples

S. Nihira and G. Chiba

Combination of sensitivity-based and random sampling-based methodologies for efficient uncertainty quantification calculations with control variates method 
(a) Case 1

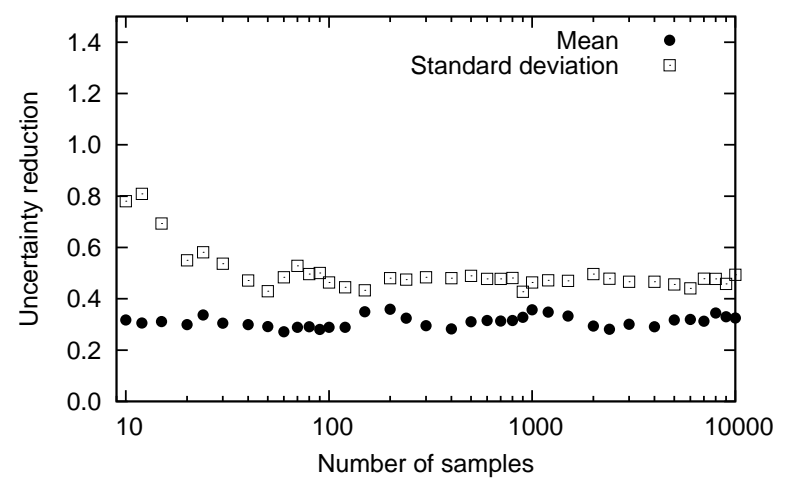

(b) Case 2

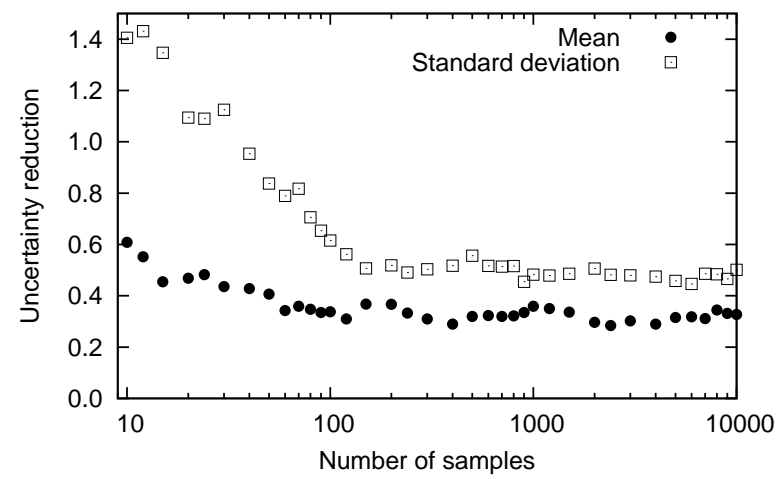

Figure 3 Uncertainty reduction in estimations of mean value and standard deviation

S. Nihira and G. Chiba

Combination of sensitivity-based and random sampling-based methodologies for efficient uncertainty quantification calculations with control variates method 
(a) Mean

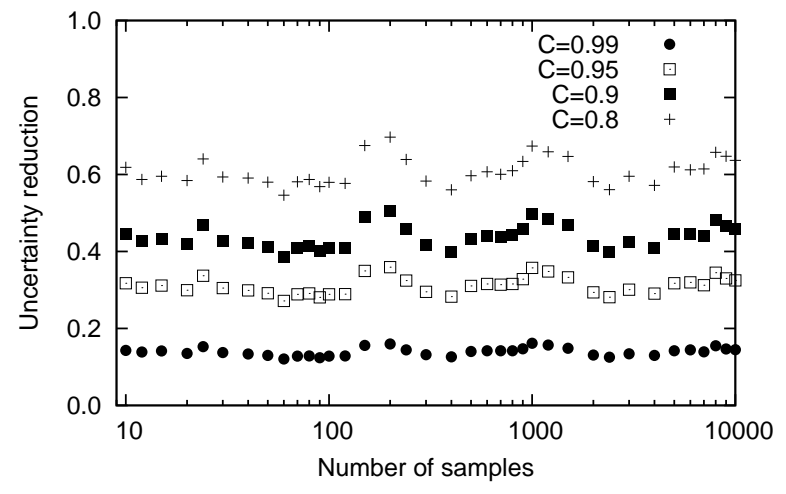

(b) Standard deviation

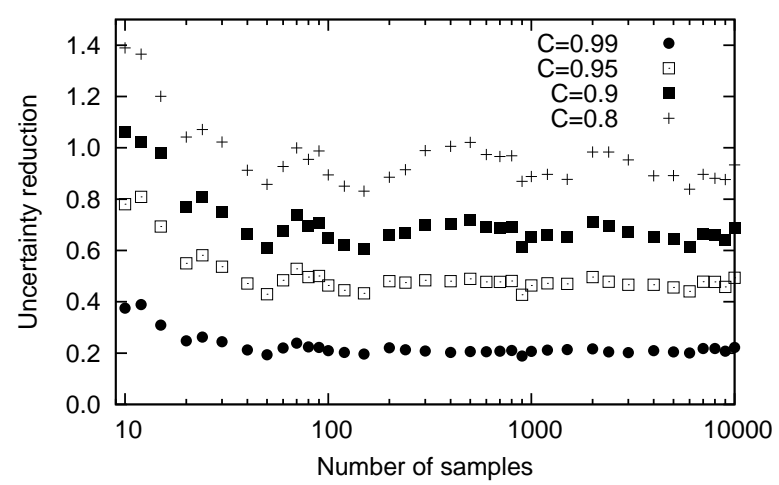

Figure 4 Dependence of uncertainty reduction in estimations of mean value and standard deviation on correlation coefficients $C$

S. Nihira and G. Chiba

Combination of sensitivity-based and random sampling-based methodologies for efficient uncertainty quantification calculations with control variates method 
(a)

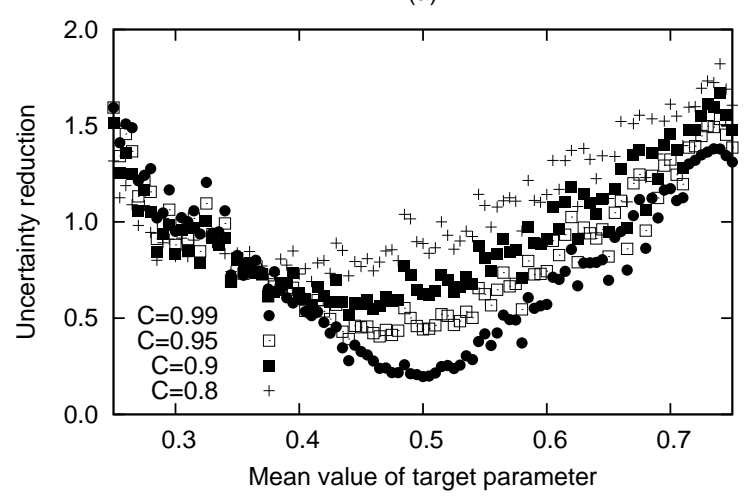

(b)

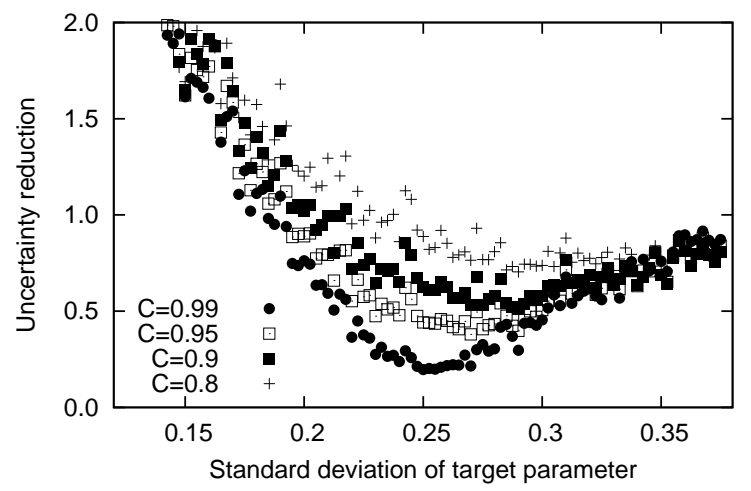

Figure 5 Uncertainty reduction in estimations of standard deviation with different conditions for a target parameter

S. Nihira and G. Chiba

Combination of sensitivity-based and random sampling-based methodologies for efficient uncertainty quantification calculations with control variates method 
(a)

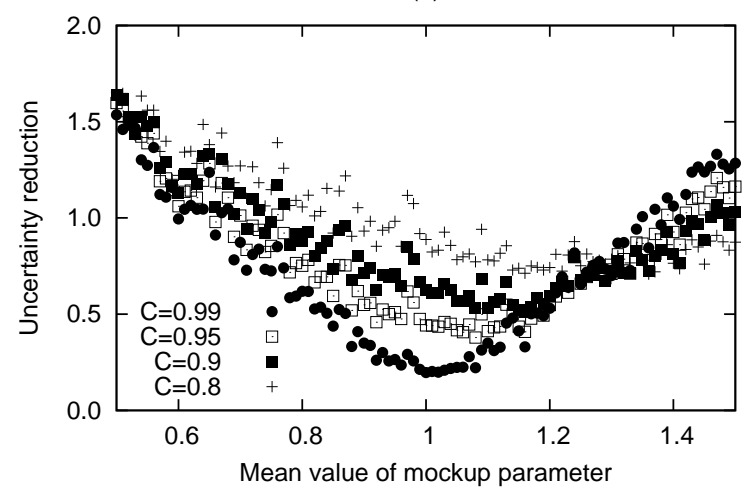

(b)

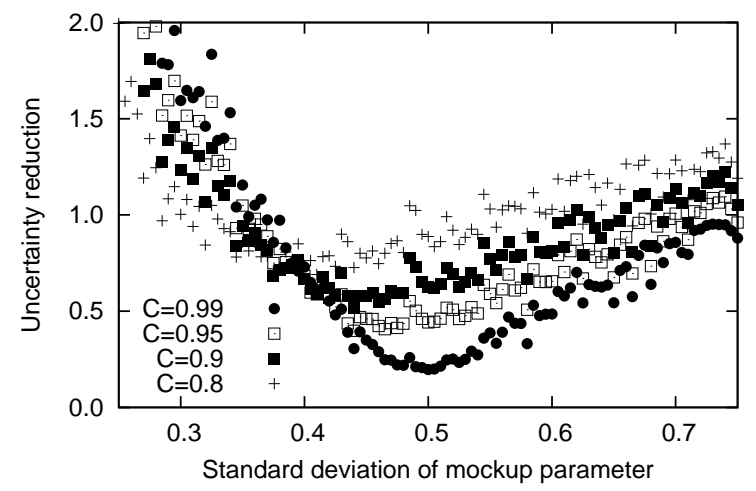

Figure 6 Uncertainty reduction in estimations of standard deviation with different conditions for a mockup parameter

S. Nihira and G. Chiba

Combination of sensitivity-based and random sampling-based methodologies for efficient uncertainty quantification calculations with control variates method 
(a) $\mathrm{C}=0.99$

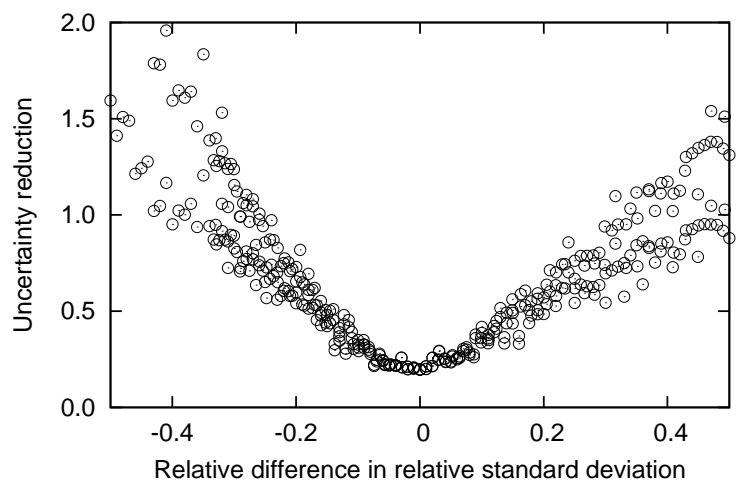

(c) $\mathrm{C}=0.9$

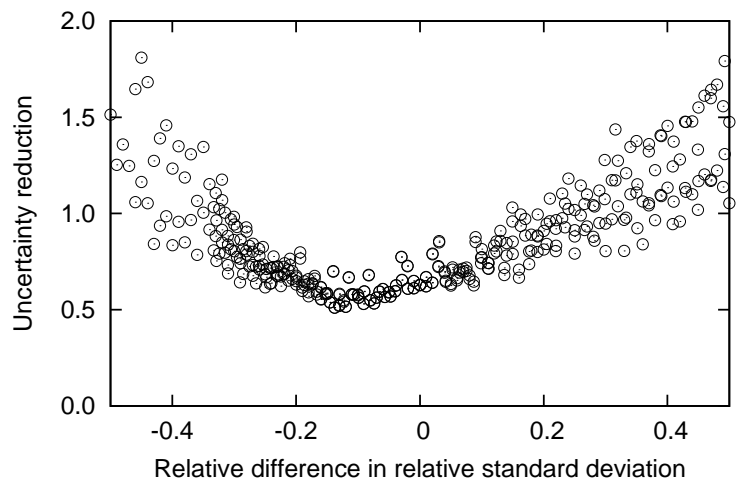

(b) $\mathrm{C}=0.95$

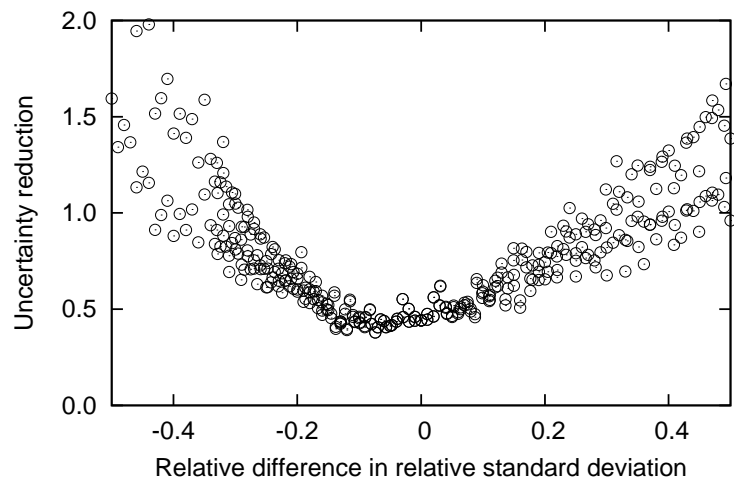

(d) $\mathrm{C}=0.8$

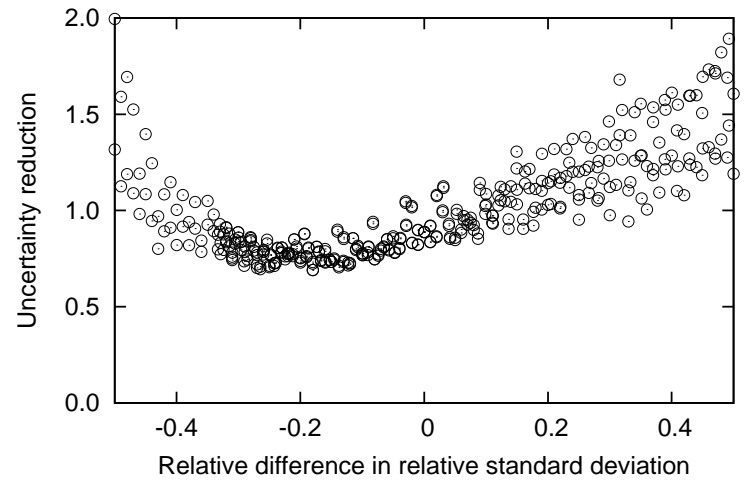

Figure 7 Uncertainty reduction in estimations of standard deviation with relative difference in relative standard deviations between target and mockup parameters

S. Nihira and G. Chiba

Combination of sensitivity-based and random sampling-based methodologies for efficient uncertainty quantification calculations with control variates method 


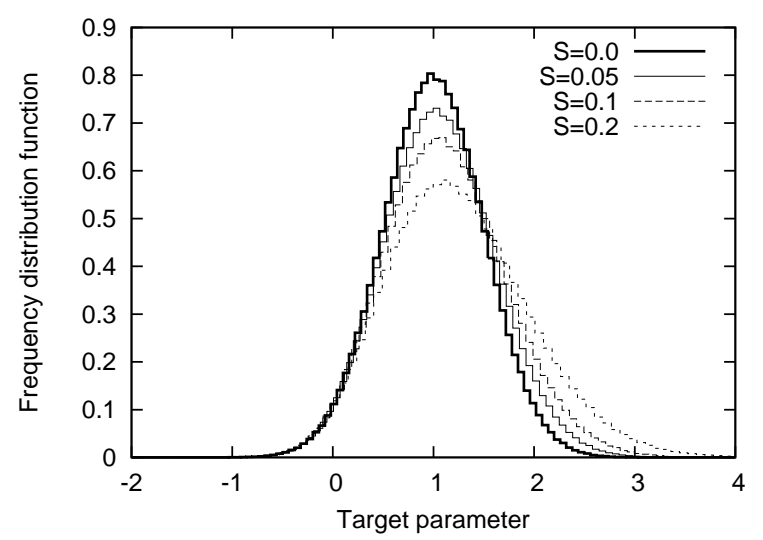

Figure 8 Frequency distribution functions of target parameters with different value of $S$

S. Nihira and G. Chiba

Combination of sensitivity-based and random sampling-based methodologies for efficient uncertainty quantification calculations with control variates method 
(a) Normal

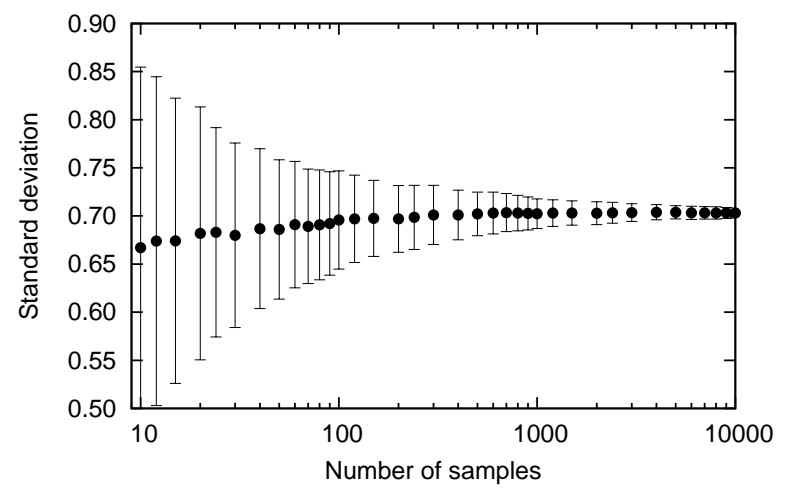

(b) $\mathrm{CV}$

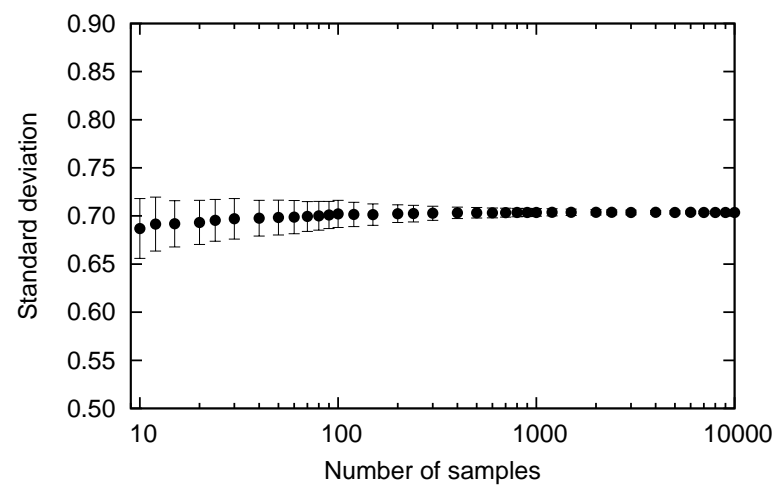

Figure 9 Standard deviations for non-linear problem in the case of $S=0.2$

S. Nihira and G. Chiba

Combination of sensitivity-based and random sampling-based methodologies for efficient uncertainty quantification calculations with control variates method 


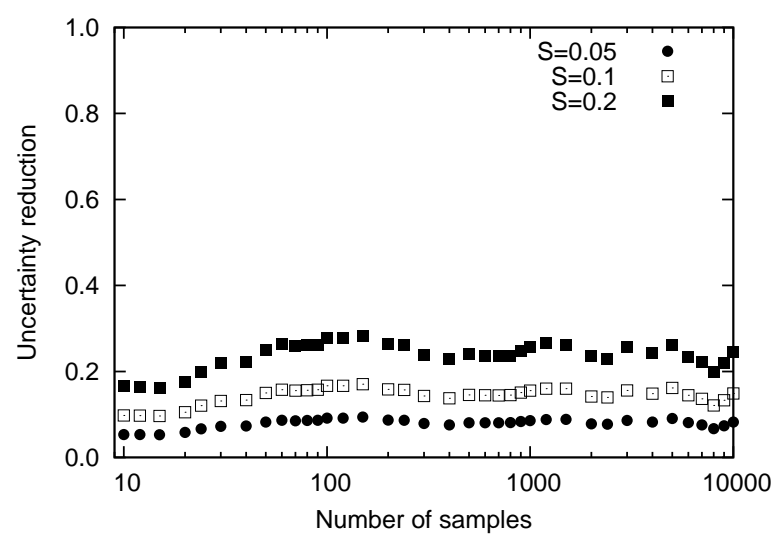

Figure 10 Uncertainty reduction of standard deviation estimated by the CV method for non-linear problem

S. Nihira and G. Chiba

Combination of sensitivity-based and random sampling-based methodologies for efficient uncertainty quantification calculations with control variates method 
(a) $\mathrm{S}=0.05$

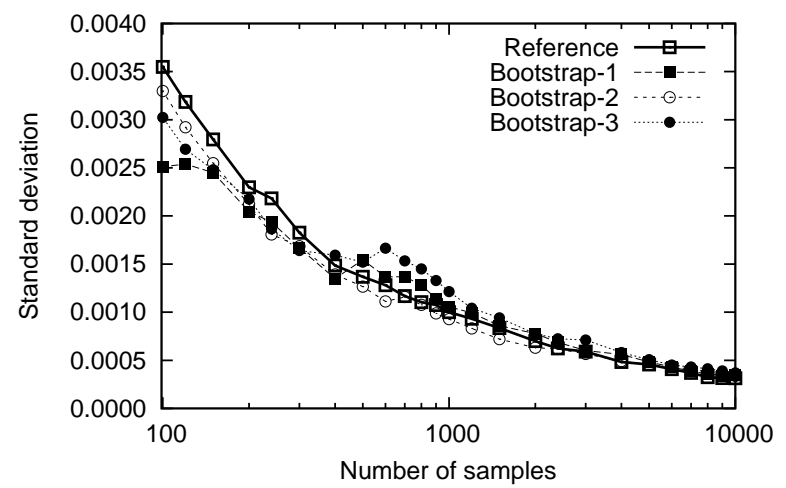

(c) $\mathrm{S}=0.2$

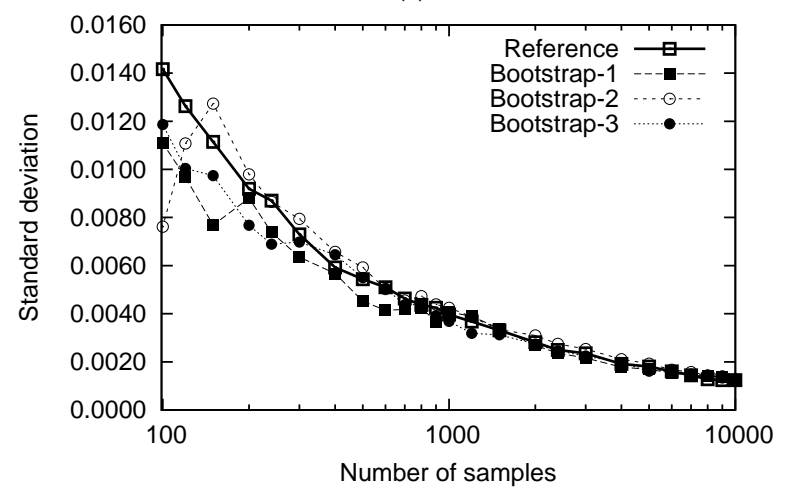

(b) $S=0.1$

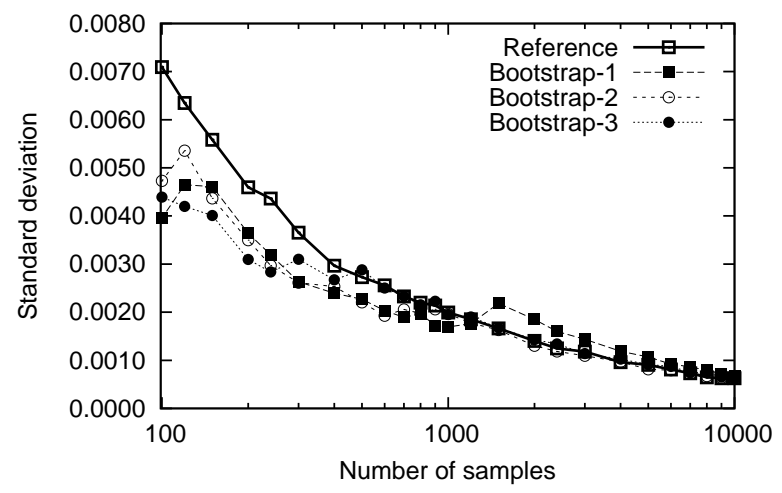

Figure 11 Standard deviation of estimated sample standard deviation calculated by the bootstrap method for non-linear system

S. Nihira and G. Chiba

Combination of sensitivity-based and random sampling-based methodologies for efficient uncertainty quantification calculations with control variates method 
(a) Normal

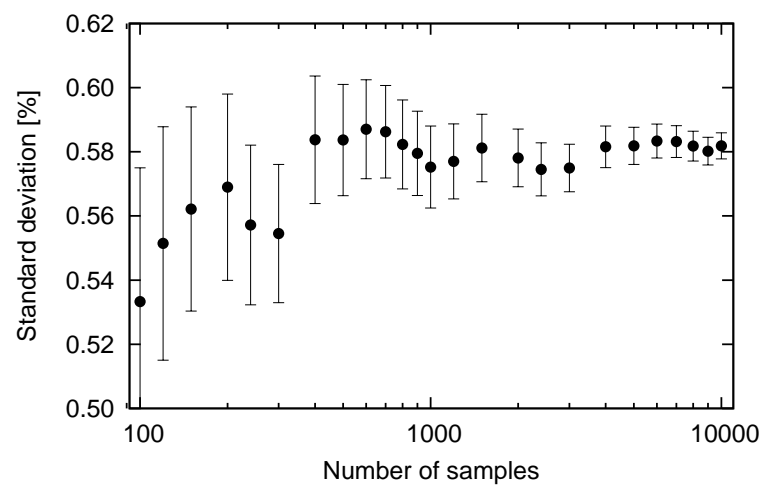

(b) $\mathrm{CV}$

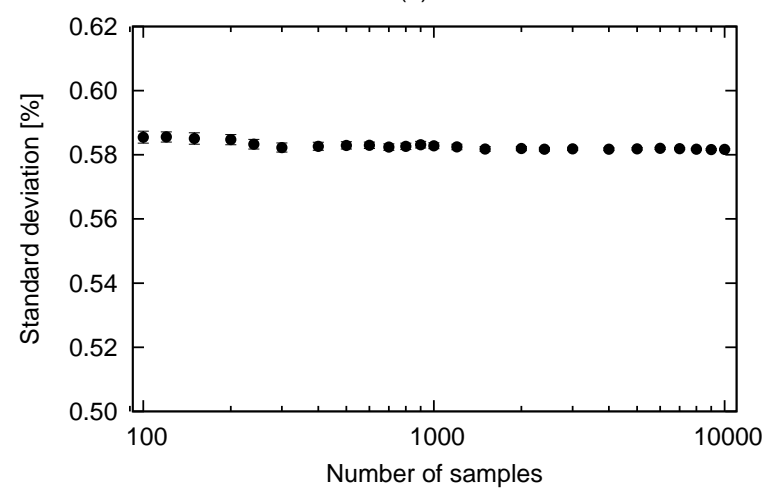

Figure 12 Standard deviation of cesium-134 number density after burnup

S. Nihira and G. Chiba

Combination of sensitivity-based and random sampling-based methodologies for efficient uncertainty quantification calculations with control variates method 
(a) Normal

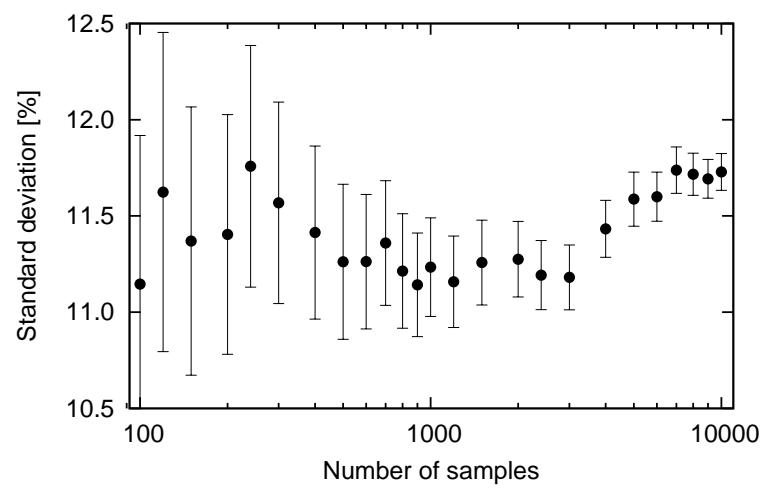

(b) $\mathrm{CV}$

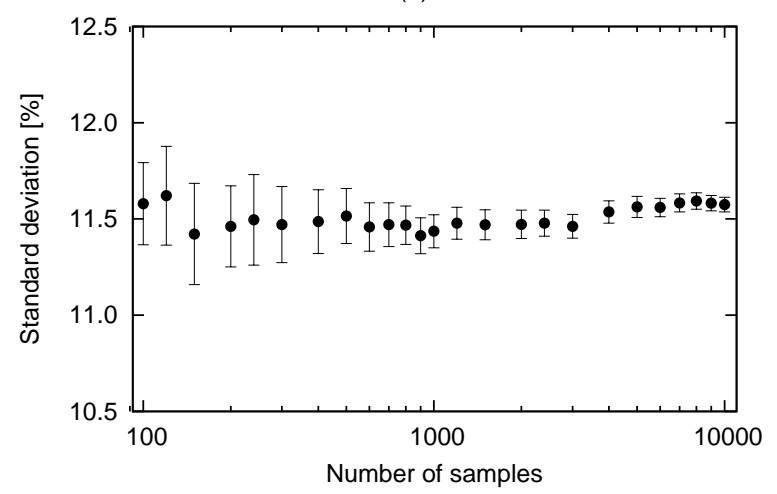

Figure 13 Standard deviation of europium-151 number density after burnup

S. Nihira and G. Chiba

Combination of sensitivity-based and random sampling-based methodologies for efficient uncertainty quantification calculations with control variates method 


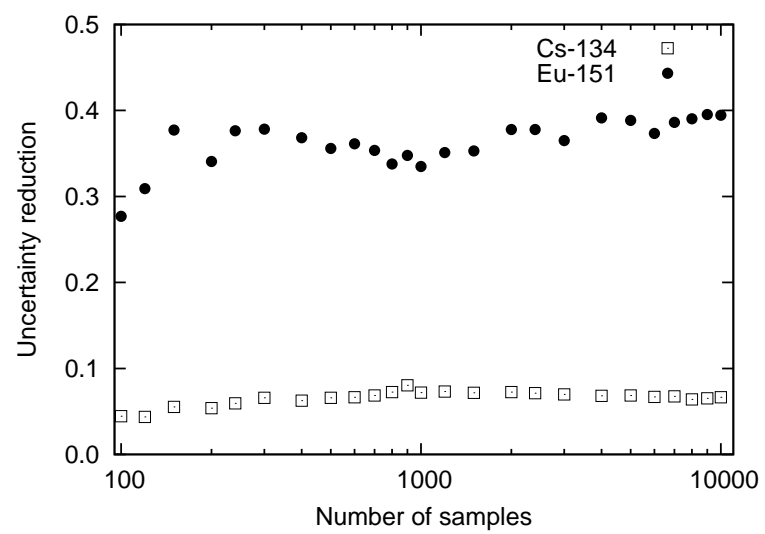

Figure 14 Uncertainty reduction in standard deviation of cesium-134 and europium-151 number density after burnup

S. Nihira and G. Chiba

Combination of sensitivity-based and random sampling-based methodologies for efficient uncertainty quantification calculations with control variates method 